\title{
Low loss GaN waveguides for visible light on Si substrates
}

\author{
M. Gromovyi \\ maksym.gromovyi@crhea.cnrs.fr
}

\section{F. Semond}

\section{J. Y. Duboz}

\section{G. Feuillet}

\section{P. De Micheli \\ marc.de-micheli@unice.fr}

CNRS, CRHEA UP 10, rue Bernard Gregory, 06560 VALBONNE, France

CNRS, CRHEA UP 10, rue Bernard Gregory, 06560 VALBONNE, France

CNRS, CRHEA UP 10, rue Bernard Gregory, 06560 VALBONNE, France

CEA LETI, 17 rue des Martyrs, 38054 Grenoble cedex 9, France

In this work, we present the fabrication and the characterization of an optical waveguide made of AIN and GaN layers grown by MBE on a $\mathrm{Si}(111)$ substrate. For the fundamental mode at $633 \mathrm{~nm}$, the propagation losses are in the order of $2 \mathrm{~dB} / \mathrm{cm}$, which is a good number for SC waveguides at this wavelength. The propagation losses dramatically increase with the mode order. A careful comparison of measurements and modeling of the complete structure allows identifying the part of the losses due to absorption in the Si substrate, and showing that propagation losses could be further reduced by using well chosen sol substrates.

[DOI: http://dx.doi.org/10.2971/jeos.2014.14050]

Keywords: GaN on silicon, GaN on SOI, waveguide losses, visible waveguide, MBE

\section{INTRODUCTIO N}

Wide bandgap group III-nitride semiconductors have interesting electrooptical properties in the near UV and the visible regions of the spectrum which have led to the successful production of LEDs and laser diodes [1]. In the past decade nonlinear properties of $\mathrm{GaN}$ became another subject for investigation. A large transparency window $350 \mathrm{~nm}-13.6 \mu \mathrm{m}$ for GaN, which can be extended up to the $200 \mathrm{~nm}$ for AlN, makes it possible to implement nonlinear processes such as sum or difference-frequency generation for the wavelength in the visible and UV regions. Second harmonic generation (SHG) based on quasi phase matching by periodical polling was demonstrated for the bulk GaN [2]. Another technique for SHG based on modal dispersion phase matching was shown in GaN planar waveguide grown by MOCVD on sapphire [3].

One important question related to frequency conversion in wavegudes is the integration with other technologies, which leads to the substrate issue. Naturally due to the mature technology and low cost, silicon is the most attractive candidate to which one would prefer to add GaN functionality. Now more and more efforts are done toward integration of $\mathrm{GaN}$ into Si technology. In spite of the promising results it still remains a challenge. On the level of fabrication, large lattice parameters and thermal expansion coefficient mismatch causes the problem of residual stress, defects and cracks. From the optical point of view $\mathrm{Si}$ is not a desired material since it absorbs in the visible and the UV. Early developments of the epitaxy (both MBE and MOCVD) of GaN on Si $[4,5]$ have allowed to solve most problems, so that many devices have been fabricated based on GaN grown on Si [6]-[10]. SHG based on modal phase matching was obtained in GaN and AlN microrings on $\mathrm{Si}$ substrate covered by $\mathrm{SiO}_{2}$ insulator [11, 12].

The future of integrated GaN photonics dramatically depends on the supply of the main building block: low loss $\mathrm{GaN}$ waveguides. At the present time there are few publications which discuss GaN based waveguides. These discussions mainly address the study of the linear or nonlinear properties of GaN. The losses are generally provided as additional information and the origin of the losses is rarely discussed. The reported losses for visible range are in order of several $\mathrm{dB} / \mathrm{cm}[13,14]$, and are lower for the telecom $1.55 \mu \mathrm{m}$ wavelength $(<1 \mathrm{~dB} / \mathrm{cm})[15,16]$. In addition, these articles present different fabrication conditions, as well as different measurement techniques which leads to a great dispersion of the experimental values.

We believe that the origin of the losses in GaN waveguides is a very important topic which deserves a separate discussion and a systematic study. Here we address the influence the waveguide design on the value of the propagation losses in the presence of a Si substrate, this study has not been discussed yet.

In this work we present a GaN on $\mathrm{AlN}$ waveguide grown by $\mathrm{MBE}$ on $\mathrm{Si}(111)$ substrate with $1.9 \mathrm{~dB} / \mathrm{cm}$ and $3.4 \mathrm{~dB} / \mathrm{cm}$ 
losses for the TE0 and TM0 modes respectively at wavelength $\lambda=632.8 \mathrm{~nm}$. Using theoretical modeling we show that the absorption in Si can significantly influence the propagation losses of the guided modes. The theoretical model presented below allows to estimate the thickness of AlN layer which is needed for optically isolating the GaN guiding layer from the absorbing Si substrate. We also show that by replacing Si substrates by properly chosen SOI substrates one can significantly reduce the losses related to the absorption in $\mathrm{Si}$ and obtain a larger flexibility on the choice of AIN buffer layer thickness.

\section{EXPERIMENT}

\subsection{Waveguide fabrication}

The sample we tested was epitxially grown by ammonia-MBE on a 2 " $\mathrm{Si}(111)$ wafer. It is composed of a $360 \mathrm{~nm}$ AlN buffer grown at $1000^{\circ} \mathrm{C}$ on top of which was grown a $1 \mu \mathrm{m}$ thick GaN layer at $800^{\circ} \mathrm{C}$. Due to a large thermal expansion coefficient mismatch between nitrides and the silicon substrate, nitrides epitaxially grown on silicon are under tensile strain at room temperature and cracks appear if the amount of tensile strain exceeds a certain value. For this sample, layer thicknesses are adjusted in order to decrease as much as possible the wafer bow indicating that the tensile strain is mostly compensated by playing with the lattice mismatch in between $\mathrm{GaN}$ and AlN.

We chose a 1 micron thick GaN layer to have enough guided modes at $633 \mathrm{~nm}$ to allow a precise characterisation of the refractive index profiles of the waveguide with the prism coupling technique. Indeed this characterisation method allows deducing refractive indices and thicknesses of the different epitaxial layers constituting the waveguide from the measurement of the outcoupling angles for the different modes. The more modes the more precision in the determination of the different parameters. Moreover this thickness is adapted for frequency doubling from $1200 \mathrm{~nm}$ to $600 \mathrm{~nm}$.

\subsection{Waveguide characterization}

We characterized the waveguide using the prism coupling technique [17] and a HeNe laser at $632.8 \mathrm{~nm}$. We used two rutile prisms: the first one for coupling the light into the waveguide, and the second one for outcoupling the light from the waveguide and observing M-lines. For $1 \mu \mathrm{m}$ thick GaN layer

\begin{tabular}{|c|c|c|}
\hline TE-modes & $\begin{array}{c}\text { Angle } \\
\left(\mathbf{}^{\circ}\right)\end{array}$ & Effective index \\
\hline TM0 & 32.278 & 2.3754 \\
\hline TM1 & 23.926 & 2.3212 \\
\hline TM2 & 12.521 & 2.2318 \\
\hline TM3 & 0.182 & 2.1245 \\
\hline TE0 & -0.719 & 2.3380 \\
\hline TE1 & -5.606 & 2.2878 \\
\hline TE2 & -13.253 & 2.2062 \\
\hline TE3 & -23.527 & 2.0935 \\
\hline
\end{tabular}

TABLE 1 Effective indices for the TE and TM modes measured by $M$-lines technique. on AlN buffer layer we were able to observe 4 TE and 4 TM modes.

Measuring the outcoupling angles of the different modes, we obtained effective indices of the propagating modes, see Table 1 . The value of the effective index for a given mode is determined by the thickness of the guiding layer, and by the refractive indices of the guiding layer and the substrate. In the case of assymmetric step-index planar waveguides, there exist a simple analytical solution which links these quantities [20]. Using these analytical relations and the 8 measured effective indices of the TE and TM modes, we solved the inverse problem and determined the values of the 5 unknown parameters: ordinary and extraordinary refractive indices of $\mathrm{GaN}$ and AlN and the waveguide thickness. The result for the refractive indices of $\mathrm{GaN}$ and AlN are presented in Table 2, these values are in a good agreement with previously obtained data $[18,19]$. As the layers are obtained by MBE the error on their thicknesses are negligible. The errors of the determined values of the GaN and AlN refractive indices are coming from the errors made during the measurement of the outcoupling angles. The amplitude of the errors is determined by injecting extreme values in the calculation.

It should be noted that our method gives much better precision for the values of refractive index of GaN than for the AlN index, since only a very small part of the electromagnetic field is confined in the AlN.

The observation shows that light is locally scattered by some defects and also is more uniformly scattered by the interface roughness. If we assume a uniform scattering rate in the measurement area, then the light intensity measured outside the sample is proportional to the light intensity within the guide for a given mode. We chose a region without any defect, and measured the light intensity at different positions along the propagation line. Since $1 \mu \mathrm{m} \mathrm{GaN} \mathrm{layer} \mathrm{on} \mathrm{AlN} \mathrm{forms}$ a multi-mode waveguide we tried to measure coupling between modes by observing M-lines with 0 -mode being exited. Selectively exciting the TE0 mode it was possible to observe some coupling in the TE1 but the measurements indicate that the intensity coupled into the TE1 mode is at least two orders of magnitude lower than the power present in the TE0 mode. The situation is identical for the TM0 and the TM1 modes, therefore in the following we will neglect this coupling. For the loss measurement we used a highly sensitive, low noise sCMOS camera and the obtained signal was very well matching an exponential decay behaviour, see Figure 1, giving the losses values $1.9 \mathrm{~dB} / \mathrm{cm}$ for the TE0 and $3.4 \mathrm{~dB} / \mathrm{cm}$ for the

\begin{tabular}{|c|c|c|}
\hline$\lambda=632.8 \mathrm{~nm}$ & $\boldsymbol{n}_{\mathbf{o}}$ & $\boldsymbol{n}_{\mathbf{e}}$ \\
\hline $\mathrm{GaN}$ & $2.354 \pm 0.001$ & $2.393 \pm 0.001$ \\
\hline $\mathrm{AlN}$ & $2.075 \pm 0.02$ & $2.117 \pm 0.02$ \\
\hline$\Delta \mathrm{n}$ & 0.279 & 0.276 \\
\hline
\end{tabular}

TABLE 2 Ordinary and extraordinary refractive indices of GaN and AIN obtained from waveguide characterization. The errors of the determined values of the GaN and AIN refractive indices are coming from the errors made during the measurement of the outcoupling angles. The amplitude of the errors is determined by injecting extreme values in the calculation. 


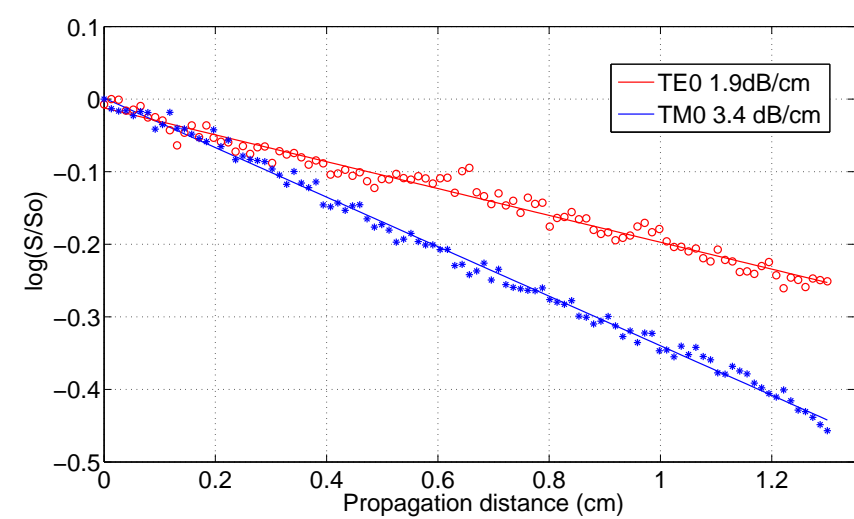

FIC. 1 Propagation loss estimation for the TEo and TMo modes by surface diffusion measurement. Vertical axis gives the logarithm on a base 10 of the ratio between diffused light intensity along the propagation line and diffused light intensity at the starting point, as a function of the propagation distance.

TM0 modes with an uncertainty of $\pm 0.1 \mathrm{~dB} / \mathrm{cm}$, the error of the linear least square approximation. The obtained values indicate that guiding layer are of good quality at this wavelength and present a good potential for device fabrication in the UV and the Visible. Selectively exciting the TE1 or TM1 modes, one can hardly observe the diffusion at the surface after $1 \mathrm{~cm}$ of propagation, this distance is even shorter for higher order modes indicating that, for these modes, the losses reach $20 \mathrm{~dB} / \mathrm{cm}$ or more.

\section{MODELLING}

\subsection{Theoretical model}

To describe the loss mechanism due to the absorption in $\mathrm{Si}$ layer, it is not enough to consider a simple three layer model (air/GaN/AlN) for which an analytical solution is known. One has to consider multilayered models which will include $\mathrm{Si}$ or SOI substrates into the equations. In the beginning we introduce a theoretical model for the general case of a multilayered plane waveguide, the scheme of which can be seen in Figure 2. We assume that different layers have different physical properties, specifically different refractive indices. Speaking about one separate layer, we are interested in the modelling of the wave propagation in uniaxial materials, such as GaN or AlN, with $\mathrm{Oz}$ axes associated with an extraordinary refractive index $n_{e}$, and the $O x y$-plane with an ordinary refractive index $n_{0}$. In our model, one given layer has homogeneous and isotropic properties in Oxy-plane, and we assume that $n_{o}$ and $n_{e}$ do not depend on $z$-coordinate within the layer. To describe the losses in the waveguide we allow refractive indices to be complex numbers.

To summarize the above description above in mathematical terms, the dielectric tensor for one layer has the following form:

$$
\hat{\varepsilon}=\varepsilon_{o}\left(\begin{array}{ccc}
n_{o}^{2} & 0 & 0 \\
0 & n_{o}^{2} & 0 \\
0 & 0 & n_{e}^{2}
\end{array}\right),
$$

where $\varepsilon_{0}$ is the vacuum permittivity, and $n_{0}, n_{e}$ are the ordinary and extraordinary refractive indices. Dielectric tensor is

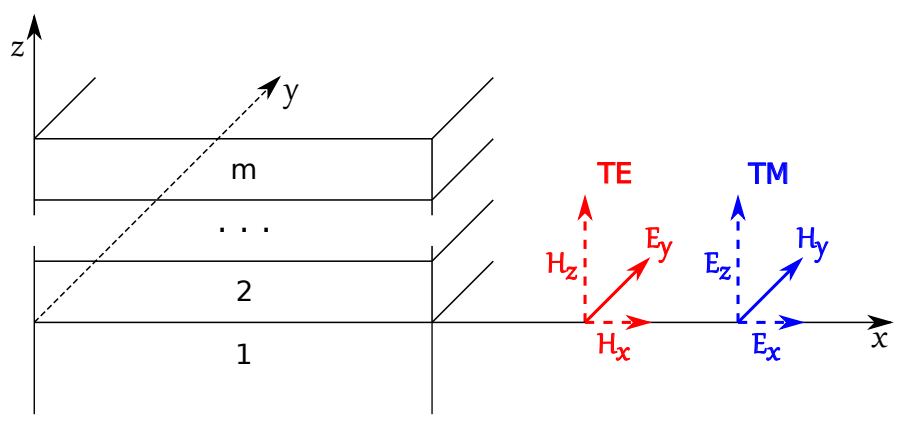

FIG. 2 General scheme of a planar multilayered waveguide. The electromagnetic field propagates in the waveguide in $x$-direction in the form of modes. The modes of the waveguide can be divided into two groups TE-modes, defined by a single $y$-component of the electric field, and TM-modes - single $y$-component of the magnetic field.

a piecewise constant function of $\mathrm{z}$-coordinate:

$$
\hat{\varepsilon}(z)=\left\{\begin{array}{cc}
\hat{\varepsilon}_{1}, & 1^{\text {st }} \text { layer }, \\
\hat{\varepsilon}_{2}, & 2^{\text {nd }} \text { layer } \\
\ldots & \ldots \\
\hat{\varepsilon}_{m}, & m^{\text {th }} \text { layer } .
\end{array}\right.
$$

As it was mentioned above the modes in the planar waveguide can be divided into two group TE and TM modes. In the coordinate system displayed on the Figure ??, TE modes are described by the next equations:

$$
\text { TE modes: } \begin{aligned}
E_{y}(x, z, t) & =\mathcal{E}_{y}(z) \exp (i \beta x-i \omega t), \\
\left.H_{x}(x, z) t\right) & =-\frac{1}{i \omega \mu_{0}} \frac{\partial E_{y}}{\partial z}, \\
H_{z}(x, z, t) & =\frac{1}{i \omega \mu_{0}} \frac{\partial E_{y}}{\partial x},
\end{aligned}
$$

where $\beta=k n$ is the propagation constant along $\mathrm{x}$-direction, $n$ is the effective refractive index of a given mode, $k=\omega / c$ is the wave vector in the vacuum.

The system of equations for the amplitude $\mathcal{E}_{y}$ will have the form:

$$
\begin{aligned}
& \mathcal{E}_{y}^{\prime \prime}(z)+k^{2}\left[n_{o}^{2}(k, z)-n^{2}\right] \mathcal{E}_{y}(z)=0, \\
& \mathcal{E}_{y}(z), \mathcal{E}_{y}^{\prime}(z) \text { - continuous, } \\
& \mathcal{E}_{y}( \pm \infty)=0,
\end{aligned}
$$

where by $\mathcal{E}_{y}^{\prime}$ and $\mathcal{E}_{y}^{\prime \prime}$ we denote first and second derivatives with respect to $z, n_{o}(k, z)$ is the ordinary refractive index, which depend on the wave vector $k$, and also a piecewise constant function of the layer with respect to $\mathrm{z}$ coordinate.

Similarly, the electric and the magnetic fields associated with the TM-modes are give by

$$
\begin{aligned}
H_{y}(x, z, t) & =\mathcal{H}_{y}(z) \exp (i \beta x-i \omega t), \\
\text { TM modes: } \quad E_{x}(x, z, t) & =\frac{1}{i \omega n_{o}^{2}} \frac{\partial H_{y}}{\partial z}, \\
E_{z}(x, z, t) & =-\frac{1}{i \omega n_{e}^{2}} \frac{\partial H_{y}}{\partial x} .
\end{aligned}
$$

where $\beta=k n$ is the propagation constant along $\mathrm{x}$-direction, $n$ is the effective refractive index of a given mode, where $k=\omega / c$ is the wave vector in the vacuum. 


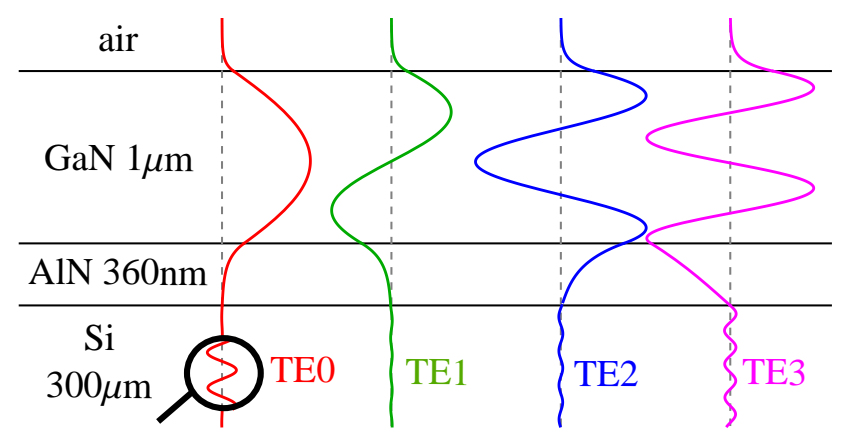

FIC. 3 TE modes profiles.

The equations for the unknown amplitude $\mathcal{H}_{y}$ :

$$
\begin{aligned}
& \mathcal{H}_{y}^{\prime \prime}(z)+k^{2} \frac{n_{o}^{2}(k, z)}{n_{e}^{2}(k, z)}\left[n_{e}^{2}(k, z)-n^{2}\right] \mathcal{H}_{y}(z)=0, \\
& \mathcal{H}_{y}(z), \frac{1}{n_{o}^{2}(k, z)} \mathcal{H}_{y}^{\prime}(z)-\text { continuous, } \\
& \mathcal{H}_{y}( \pm \infty)=0 .
\end{aligned}
$$

Eqs. (4) and (6) represent an eigenvalue problems solving which one can find effective indices of TE and TM modes respectively. Knowing these effective indices we can estimate the propagation losses. The losses are characterized either by the absorption coefficient $\alpha$ or by propagation losses $L$ :

$$
\alpha=\frac{4 \pi}{\lambda} \operatorname{Im}(n)\left[\mathrm{cm}^{-1}\right], \quad L=4.3 \alpha\left[\mathrm{dB} \mathrm{cm}^{-1}\right],
$$

where $\lambda$ is the wavelength, $\operatorname{Im}(n)$ - imaginary par of the refractive index of a given mode.

\subsection{Si substrate}

To calculate the losses due to the absorption in the Si substrate we consider a 5 layer system: air/GaN/AlN/Si/air. The main advantage of the 5 layers model is that the index of the external layers (air) is smaller than the effective indices of the modes we are interested in. This assures that in these layers the fields are evanescent and therefore decay to zero before reaching the edge of the calculation window.

The Si substrate is the only one presenting complex refractive index. The numerical values for the refractive indices at $\lambda=632.8 \mathrm{~nm}$ used in the calculations are taken from Table 2 for AlN and GaN and from [21] for Si. Solving the eigenvalue problems Eqs. (4) and (6) for multilayered systems is a rather technical procedure, we performed using the Mathematica package.

The results of calculations, namely the modes profiles, the modes confinement factors and the losses are presented below. Figure 3 shows the profiles of the TE modes, in addition we calculated the modes confinement factors $\eta_{\mathrm{GaN}}$ and $\eta_{\mathrm{Si}}$ :

$$
\eta_{\mathrm{GaN}}=\frac{\text { Energy in GaN layer }}{\text { Total modal energy }}, \quad \eta_{\mathrm{Si}}=\frac{\text { Energy in Si layer }}{\text { Total modal energy }},
$$

which are listed in Table 3.

Using the formulas Eq. (7) we estimated the losses due to the mode energy absorption in Si layer, results are also presented in Table 3. The errors range for the modelled losses in Table 3

\begin{tabular}{|c|c|c|c|c|}
\hline \multirow{4}{*}{ TE } & mode 0 & 99.5 & 0.005 & $1.2 \pm 0.3$ \\
\cline { 2 - 5 } & mode 1 & 97.6 & 0.034 & $9 \pm 2$ \\
\cline { 2 - 5 } & mo $)$ & $\begin{array}{c}\mathrm{L} \\
(\mathrm{dB} / \mathrm{cm})\end{array}$ \\
\cline { 2 - 5 } & mode 2 & 93.7 & 0.21 & $60 \pm 15$ \\
\cline { 2 - 5 } & mode 3 & 83.6 & 1.67 & $550 \pm 150$ \\
\hline \multirow{4}{*}{ TM } & mode 0 & 99.5 & 0.009 & $2.4 \pm 0.9$ \\
\cline { 2 - 5 } & mode 1 & 97.7 & 0.08 & $24 \pm 9$ \\
\cline { 2 - 5 } & mode 2 & 92.0 & 0.77 & $250 \pm 100$ \\
\cline { 2 - 5 } & mode 3 & 68.6 & 11.2 & $>10^{3}$ \\
\hline
\end{tabular}

TABLE 3 Results of modeling: the confinement factors $\eta_{\mathrm{GaN}}, \eta_{\mathrm{Si}}$ and the losses $L$ caused by the absorption in the Si substrate for the different TE and TM modes.

\begin{tabular}{|c|c|c|}
\hline$\lambda=632.8 \mathrm{~nm}$ & TE0 & TM0 \\
\hline Measured total losses $(\mathrm{dB} / \mathrm{cm})$ & $1.9 \pm 0.1$ & $3.4 \pm 0.1$ \\
\hline Modelled absorption in Si $(\mathrm{dB} / \mathrm{cm})$ & $1.2 \pm 0.3$ & $2.4 \pm 0.9$ \\
\hline$\Delta \mathrm{dB} / \mathrm{cm}$ & $0.7 \pm 0.4$ & $1.0 \pm 1.0$ \\
\hline
\end{tabular}

TABLE 4 Comparison of the total propagation losses measured during the waveguide characterization with the modeled absorption in the Si substrate.

was determined performing the calculations with the extreme values of the refractive indices from the Table 2.

The results of modelling presented in Table 3 show a natural correlation between the losses and the modal energy confinement in the Si layer. The modelling shows that the propagation losses dramatically change with the mode order which corresponds to what have been observed experimentally.

At this point it is necessary to recall that measured values represent total losses while we calculate only the absorption in the $\mathrm{Si}$, therefore the difference between these values should represent the influence of the other loss mechanisms: scattering and absorption in the other layers. In Table 4 we can see that TE0 and TM0 modes present quite different propagation losses while the difference between measured and calculated values are nearly equal. This indicates that the loss variation with polarization is mainly due to the difference of absorption in the Si substrate which directly depends on the confinement of two modes. This also indicates that losses due to other mechanisms are in the order of $1 \mathrm{~dB} / \mathrm{cm}$.

One of the possibilities to reduce the losses due to the absorption in the Si layer is to increase the AlN buffer layer thickness. Figure 4 shows which thickness of AlN is required to make absorption losses in Si negligible compared to losses due to other mechanisms.

This solution has the disadvantage of inducing very long fabrication processes since with $\mathrm{MBE}$ the speed of growth of AlN is only $0.1 \mu \mathrm{m} / \mathrm{h}$.

\subsection{SOl substrate}

The second way to reduce the losses is to use SOI substrates. They exist with different designs allowing to reduce the absorption losses by reducing the Si thickness and therefore 


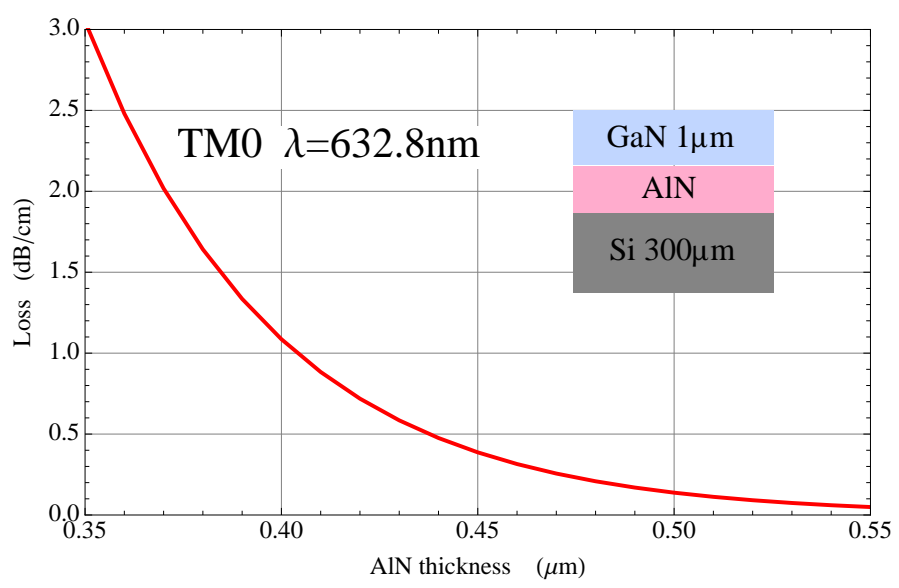

FIC. 4 Modelled absorption for the TMo mode in the Si substrate as a function of the thickness of the AIN buffer layer.

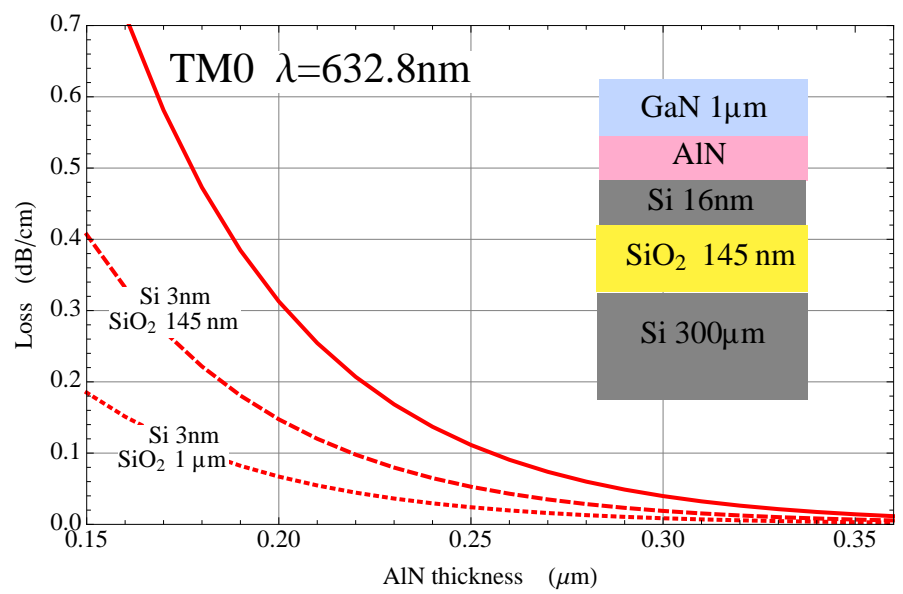

FIG. 5 Losses due to the absorption in the Si layers of SOl substrate for TMo mode as a function of the thickness of the AIN buffer layer. The solid line corresponds to the structure schematically show on the right part of the figure. Dotted lines represent possible improvement due to the functional si layer reduction and increasing the thickness of the $\mathrm{SiO}_{2}$ layer.

the overlap between the evanescent tail of the mode and the strongly absorbing medium. Figure 5 demonstrates what can be obtained with different geometries. It should be noted that we cannot eliminate the Si functional layer completely, because it is needed for the epitaxy. Ideally we would like to use SOI with thin $(<10 \mathrm{~nm}) \mathrm{Si}$ layer and thick $(>1 \mu \mathrm{m}) \mathrm{SiO}_{2}$ layer. Optimum SOI wafers are not available today, studies are under process to realise them, they will determine the technological limit that we will have to take into account for the realisation of actual devices. Nevertheless, even the structures like $\mathrm{Si}(16 \mathrm{~nm}) / \mathrm{SiO}_{2}(145 \mathrm{~nm})$ see Figure 5, which is commercially available, can significantly reduce the losses due to the absorption in the Si and allow a larger flexibility on the thickness of the AlN buffer layer.

\section{CONCLUSIONS}

In this work we present a GaN on AlN waveguide grown by MBE on a Si(111) substrate. We fully characterized this waveguide in the visible $(\lambda=632.8 \mathrm{~nm})$ by measuring its index profile using the prism coupling technique and the propagation losses of the TE and the TM fundamental modes visualizing the decrease of the scattered light along the propagation line. We obtained values of $1.9 \mathrm{~dB} / \mathrm{cm}$ and $3.4 \mathrm{~dB} / \mathrm{cm}$ respectively.
We also performed a carreful modeling of this waveguide, taking into account the full structure and the complex value of the refractive index of the $\mathrm{Si}$ substrate at this wavelengh. The comparison between the measured and the calculated values allows to deduce that the difference between the propagation losses of the TEO and the TM0 modes is perfectly explained by the difference of mode confinement and that the other loss mecanisms are responsible for approximately $1 \mathrm{~dB} / \mathrm{cm}$ losses.

Moreover the modelling allows proposing two possibilities to make the absorption losses negligible compared to the losses induced by other mechanisms: increasing the AlN buffer layer thickness, or using a carrefully chosen SOI substrate.

\section{ACKNOWLEDGEMENTS}

We acknowledge support from GANEX (ANR-11-LABX0014). GANEX belongs to the public funded "Investissements d'Avenir" program managed by the French ANR agency.

\section{References}

[1] S. Nakamura, and G. Fasol, The blue laser diode: GaN based light emitters and lasers (Springer, Berlin, 1997).

[2] A. Chowdhury, H. M. Ng, M. Bhardwaj, and N. G. Weimann, "Second-harmonic generation in periodically poled CaN," Appl. Phys. Lett. 83, 1077-1079 (2003).

[3] D. N. Hahn, G. T. Kiehne, J. B. Ketterson, G. K. L. Wong, P. Kung, A. Saxler, and M. Razeghi, "Phase-matched optical secondharmonic generation in GaN and AIN slab waveguides," J. Appl. Phys. 85, 2497-2500 (1999).

[4] F. Semond, Y. Cordier, N. Grandjean, F. Natali, B. Damilano, S. Vézian, and J. Massies. "Molecular beam epitaxy of group-III nitrides on silicon substrates: growth, properties and devices applications," Phys. Status Solidi A 188, 501-510 (2001).

[5] E. Feltin, B. Beaumont, M. Laugt, P. De Mierry, P. Vennegues, H. Lahreche, M. Leroux, et al., "Stress control in GaN grown on silicon (111) by metalorganic vapor phase epitaxy," Appl. Phys. Lett. 79, 3220 (2001).

[6] J. Y. Duboz, J. L. Reverchon, D. Adam, B. Damilano, F. Semond, N. Grandjean, and J. Massies "High performance solar blind detectors based on AlGaN grown by MBE on Si," Phys. Status Solidi A 188, 325-328 (2001).

[7] F. Semond, P. Lorenzini, N. Grandjean, and J. Massies“High electron mobility AlGaN/GaN heterostructures grown on $\mathrm{Si}(111)$ by molecular beam epitaxy," Appl. Phys. Lett. 82, 335 (2001).

[8] S. Dalmasso, E. Feltin, P. De Mierry, B. Beaumont, P. Gibart, and M. Leroux, "Green electroluminescent (Ga,InAI)N LEDs grown on Si(111)," Electron. Lett. 36, 1728-1730 (2000).

[9] A. Krost, and A. Dadgar "GaN-Based Devices on Si," Phys. Status Solidi A 194, 361-375, (2002).

[10] E. V. Lutsenko, V. N. Pavlovskii, V. Z. Zubialevich, A. I. Stognij, A. L. Gurskii, and V. A. Hryshanau, "Growth, stimulated emission, photoand electroluminescence of InGaN/GaN EL-test heterostructures," Phys. Status Solidi A 0, 272-275 (2002).

[11] C. Xiong, W. Pernice, K. K. Ryu, C. Schuck, K. Y. Fong, T. Palacios, and H. X. Tang "Integrated GaN photonic circuits on silicon (100) for second harmonic generation," Opt. Express 19, 10462-10470 (2011). 
[12] W. H. P. Pernice, C. Xiong, C. Schuck, and H. X. Tang "Second harmonic generation in phase matched aluminum nitride waveguides and micro-ring resonators," Appl. Phys. Lett. 100, 223501 (2012).

[13] E. Doghèche, D. Rémiens, A. Boudrioua, and J. C. Loulergue "Growth and optical characterization of aluminum nitride thin films deposited on silicon by radio-frequency sputtering," Appl. Phys. Lett. 74, 1209 (1999).

[14] H. P. D. Schenk, E. Feltin, M. Laügt, 0. Tottereau, P. Vennéguès, and $E$. Doghèche "Realization of waveguiding epitaxial GaN layers on Si by low-pressure metalorganic vapor phase epitaxy," Appl. Phys. Lett. 83, 5139 (2003).

[15] R. Geiss, A. Chowdhury, C. M. Staus, H. M. Ng, S. S. Park, and J. Y. Han, "Low loss GaN at 1550 nm," Appl. Phys. Lett. 87, 132107 (2005).

[16] A. Stolz, E. Cho, E. Doghèche, Y. Androussi, D. Troadec, D. Pavlidis, and D. Decoster "Optical waveguide loss minimized into gallium nitride based structures grown by metal organic vapor phase epitaxy," Appl. Phys. Lett. 98, 161903 (2011).
[17] P. K. Tien, and R. Ulrich, "Theory of prism-film coupler and thinfilm light guides," J. Opt. Soc. Am. 60, 1325-1337 (1970).

[18] U. Ozgur, G. Webb-Wood, H. O. Everitt, F. Yun, and H. Morkoc, "Systematic measurement of $\mathrm{Al}_{\mathrm{X}} \mathrm{Ca}_{1-\mathrm{x}} \mathrm{N}$ refractive indices," Appl. Phys. Lett. 79, 4103-4105 (2001).

[19] S. Pezzagna, J. Brault, M. Leroux, J. Massies, and M. de Micheli, "Refractive indices and elasto-optic coefficients of GaN studied by optical waveguiding," J. Appl. Phys. 103, 123112 (2008).

[20] A. Yariv, Quantum Electronics (Wiley, New York, 1975).

[21] E. D. Palik (ed.), Handbook of optical constants of solids (Academic Press, San Diego, 1998). 